\title{
Can Body Mass Index Predict the Difficulty of Colonoscopy?
}

\author{
Eui Joo Kim and Yoon Jae Kim \\ Division of Gastroenterology, Department of Internal Medicine, Gachon University Gil Medical Center, Gachon University College of \\ Medicine, Incheon, Korea
}

See "Obesity and Cecal Intubation Time" by Deepanshu Jain, Abhinav Goyal and Jorge Uribe, on page 187-190.

Colonoscopy has been widely used as a screening modality for colorectal cancer and in the treatment of colon disorders. Generally, colonoscopy is safe, well tolerated, and recognized as a standard diagnostic and treatment method for colon disorders. Cecal intubation (CI) is one of the proposed quality indicators for colonoscopy. ${ }^{1}$ In screening patients, the reported CI rate was $\geq 97 \%$. ${ }^{2,3}$ In other studies, CI was reported to be influenced by advanced age, female sex, history of abdominal surgery, diverticulosis, colonoscopic experience of the endoscopist, and poor bowel preparation. ${ }^{4-7}$

In this issue of Clinical Endoscopy, Jain et al. ${ }^{8}$ reported that body mass index (BMI) had a positive association with $\mathrm{CI}$ time in women, but a negative association in men. The mean CI time (MCT) was 15.9 \pm 7.9 minutes for the men and 15.5 \pm 7.9 minutes for the women. The MCTs across the BMI categories were as follows: category I ( $<24.9$ minutes), $14.4 \pm 6.5$ and $16.3 \pm 8.9$ minutes; category II (25 to 29.9 minutes), $15.5 \pm 8.3$ and $15.9 \pm 8.0$ minutes; and category III ( $\geq 30$ minutes), $16.2 \pm 8.1$ and $15.6 \pm 7.2$ minutes for women and men, respectively. However, the differences across BMI categories I, II, and III in each gender were not statistically significant ( $p=0.55$ and $p=0.95$, respectively) and just showed the trend in each

Received: February 25, 2016 Accepted: March 4, 2016

Correspondence: Yoon Jae Kim

Division of Gastroenterology, Department of Internal Medicine, Gachon University Gil Medical Center, Gachon University College of Medicine, 21 Namdong-daero 774beon-gil, Namdong-gu, Incheon 21565, Korea

Tel: +82-32-460-3778, Fax: +82-32-460-3408, E-mail: yoonmed@gachon.ac.kr

(c) This is an Open Access article distributed under the terms of the Creative Commons Attribution Non-Commercial License (http://creativecommons.org/ licenses/by-nc/3.0) which permits unrestricted non-commercial use, distribution, and reproduction in any medium, provided the original work is properly cited. sex. Jain et al. ${ }^{8}$ suggested the possible reason for the difference in the site of fat accumulation between the men and women. This is a unique point of this study. The men were more prone to accumulate fat in the abdomen, whereas the women were more prone to accumulate fat in the gluteal region and limbs than in the abdomen.

To control the variables, this study excluded patients who had poor bowel preparation, had a history of abdominal/ pelvic surgery, and who had procedure performed by a fellow. However, other variables such as constipation and visceral fat were not analyzed in this study.

Nagata et al. ${ }^{7}$ reported that female sex, lower BMI, smaller visceral adipose tissue area, smaller subcutaneous adipose tissue area, higher constipation score, history of abdominal surgery, poor bowel preparation, and examination by a fellow were independently associated with longer insertion time. Nagata et al. ${ }^{7}$ used computed tomography to evaluate visceral fat. This study demonstrated that female sex and visceral fat were independent risk factors of prolonged CI time.

The present study by Jain et al. ${ }^{8}$ has several interesting points. It demonstrates a reliable association between obesity and difficult colonoscopy across both sexes. These results could help in the prediction of accurate colonoscopy time.

However, the results of this study should be interpreted cautiously because poor bowel preparation and sex were also reported to be related with BMI and high BMI was related to suboptimal bowel preparation. ${ }^{9}$ Exclusion of patients with poor bowel preparation could be a possible reason of selection bias. Although the authors suggested a possible mechanism of the sex difference, this was not confirmed in the study and the result was not statistically significant. This was a retrospective 
single-center study.

Various approaches have been proposed to improve the quality of colonoscopy. Prediction of incomplete colonoscopy examination and improvement of the quality of colonoscopy are highly important in the diagnosis and treatment of colorectal disease. The results of the present study might help prevent delays and improve workflow within the endoscopy unit.

\section{Conflicts of Interest}

The authors have no financial conflicts of interest.

\section{REFERENCES}

1. Rex DK, Schoenfeld PS, Cohen J, et al. Quality indicators for colonoscopy. Gastrointest Endosc 2015;81:31-53.

2. Rathgaber SW, Wick TM. Colonoscopy completion and complication rates in a community gastroenterology practice. Gastrointest Endosc 2006;64:556-562.

3. Schoenfeld P, Cash B, Flood A, et al. Colonoscopic screening of average-risk women for colorectal neoplasia. N Engl J Med 2005;352:20612068.

4. Anderson JC, Gonzalez JD, Messina CR, Pollack BJ. Factors that predict incomplete colonoscopy: thinner is not always better. Am J Gastroenterol 2000;95:2784-2787.

5. Krishnan P, Sofi AA, Dempsey R, Alaradi O, Nawras A. Body mass index predicts cecal insertion time: the higher, the better. Dig Endosc 2012;24:439-442.

6. Anderson JC, Messina CR, Cohn W, et al. Factors predictive of difficult colonoscopy. Gastrointest Endosc 2001;54:558-562.

7. Nagata N, Sakamoto K, Arai T, et al. Predictors for cecal insertion time: the impact of abdominal visceral fat measured by computed tomography. Dis Colon Rectum 2014;57:1213-1219.

8. Jain D, Goyal A, Uribe J. Obesity and cecal intubation time. Clin Endosc 2016;49:187-190.

9. Parra Pérez VF, Watanabe Yamamoto J, Nago Nago A, et al. Factors related to a suboptimal bowel preparation for colonoscopy. Acta Gastroenterol Latinoam 2015;45:24-30. 